\title{
La evaluación docente. Una herramienta en la mejora del proceso enseñanza- aprendizaje en las instituciones de educación superior
}

\section{The teaching evaluation. A tool in improving the teaching-learning process in higher education institutions}

GAMBOA-CERDA, Silvia*†, VILLEGAS-FLORES, Ignacio, MEDELLÍN-RAMÍREZ, Juan Emilio y HERRERA-CHAIRES, María del Carmen

Facultad de Contaduría y Administración, Universidad Autónoma de San Luis Potosí, Madero No. 849, C. P. 78000, Centro, San Luis Potosí, S. L. P.

ID $1^{\text {er }}$ Autor: Silvia, Gamboa-Cerda / ORC ID: 0000-0002-2555-3918, Researcher ID: X-8584-2018, CVU CONACYT ID: 323123

ID $1^{\text {er }}$ Coautor: Ignacio, Villegas-Flores / ORC ID: 0000-0003-4672-2044, Researcher ID: X-4085-2019, CVU CONACYT ID: 642904

ID $2^{\text {do }}$ Coautor: Juan Emilio, Medellín-Ramírez / ORC ID: 0000-0003-4634-9913, Researcher ID: X-8791-2018, CVU CONACYT ID: 954329

ID $3^{\text {er }}$ Coautor: María del Carmen, Herrera-Chaires / ORC ID: 0000-0003-2666-2152, Researcher ID: X-7963-2018, CVU CONACYT ID: 380993

\section{Resumen}

Dada la importancia actual que tiene el papel de la evaluación docente en las instituciones de educación superior, surge la necesidad de conocer la percepción actual que tienen los alumnos de sus maestros y usarla como herramienta de referencia para la toma de decisiones que impacten positivamente en las prácticas del proceso enseñanzaaprendizaje, por lo que esta investigación permite conocer las fortalezas y áreas de oportunidad de los docentes de las materias de contabilidad básica de la licenciatura de Contador Público que ofrece la Facultad de Contaduría y Administración de la Universidad Autónoma de San Luis Potosí y sea utilizada en un primer momento como retroalimentación para que los docentes hagan consciente su actuar frente al grupo y por otro lado sirva a la institución como instrumento en el diseño de acciones que mejoren el desempeño de los maestros en su labor y así lograr una formación cualitativa de profesionales del área contable. Por último esta investigación sienta un precedente para que tanto la UASLP como otras instituciones de educación superior analicen y mejoren las prácticas educativas las licenciaturas que ofrecen.

Evaluación docente, Proceso enseñanza-aprendizaje, Formación cualitativa

\begin{abstract}
Given the current importance of the role of teacher evaluation in higher education institutions, there is a need to know the current perception that students have of their teachers and use it as a reference tool for decision-making that positively impacts practices of the teaching-learning process, so this research allows to know the strengths and areas of opportunity of teachers of the basic accounting subjects of the Public Accountant degree offered by the Facultad de Contaduría y Administración of the Universidad Autónoma de San Luis Potosí and be used at first as feedback so that teachers make their actions conscious in front of the group and on the other hand serve the institution as an instrument in the design of actions that improve the performance of teachers in their work and thus achieve a Qualitative training of professionals in the accounting area. Finally, this research sets a precedent for both the UASLP and other institutions of higher education to analyze and improve the educational practices of the degrees they offer.
\end{abstract}

Teacher evaluation, Teaching-learning process, Qualitative training

Citación: GAMBOA-CERDA, Silvia, VILLEGAS-FLORES, Ignacio, MEDELLÍN-RAMÍREZ, Juan Emilio y HERRERACHAIRES, María del Carmen. La evaluación docente. Una herramienta en la mejora del proceso enseñanza-aprendizaje en las instituciones de educación superior. Revista Teoría Educativa. 2019. 3-8: 1-9.

\footnotetext{
* Correspondencia del Autor (correo electrónico: silviag@uaslp.mx)

$\dagger$ Investigador contribuyendo como primer autor.
} 


\section{Introducción}

La Facultad de Contaduría y Administración de la Universidad Autónoma de San Luis Potosí revisa y actualiza de forma continua los planes de estudio de las diferentes licenciaturas que ofrece.

Para la carrera de Contador Público el plan de estudios vigente es el 2015 el cual va de la mano con el Plan Institucional de Desarrollo 2013-2023 y la visión 2023 de la propia facultad, dentro de los cuales se establece la misión de la Universidad, los valores y los 27 principios de la acción universitaria que orientan las funciones de docencia, investigación, preservación y difusión de la cultura y la gestión.

La estructura curricular basada en prácticas interdisciplinarias que se orientas ara establecer las normas, los principios y las técnicas contables establecidas a nivel nacional e internacional para el diseño, implementación, control y manejo de los sistemas de información financiera en las organizaciones para un adecuado proceso de toma de decisiones y para asegurar a la sociedad que la información económica presentada por las empresas e instituciones es real, el proceso didáctico con enfoque de competencias y desempeños, la participación activa por parte del estudiante que obligan a reorientar la utilización de los métodos pedagógicos, guiar al alumno con un enfoque interdisciplinario, dinámico e integral, por lo que el profesor asume un papel de diseñador, mediador y facilitador y conduzca a los alumnos hacia un aprendizaje colaborativo.

Para la institución resulta importante el conocer a través de instrumentos de evaluación sistemáticos la percepción que tienen los alumnos de sus docentes, conocer sus fortalezas y áreas de oportunidad, el cumplimiento adecuado de los planes de estudio y las herramientas didácticas y tecnológicas que utilizan para transmitir su conocimiento.

Por este motivo la evaluación docente que realizan los alumnos de sus maestros se convierte una herramienta imprescindible para la mejora continua del proceso educativo.

\section{Justificación}

Esta investigación permite conocer las fortalezas y áreas de oportunidad de los docentes de las materias de contabilidad básica de la licenciatura de Contador Público que ofrece la Facultad de Contaduría y Administración de la Universidad Autónoma de San Luis Potosí basado en la percepción actual que tienen los alumnos de ellos a través de la herramienta de evaluación docente que ofrece la institución y las acciones desde la óptica de los autores que se deben tomar para mejorar el desempeño de los maestros en su labor.

\section{Problema}

Se detecta que el correcto desempeño académico del alumno en las materias de profundización de la licenciatura de Contador Público que ofrece la Facultad de Contaduría y Administración de la Universidad Autónoma de San Luis Potosí dependerá del docente con quien el alumno haya tomado las materias de contabilidad básica, por lo que se pretende identificar cuáles son las competencias generales y específicas de los docentes de estas materias que los estudiantes valúan más altamente para que sean reforzadas en todos los docentes y con ello se mejore el proceso enseñanza-aprendizaje y se egresen profesionales contables mejor preparados

\section{Hipótesis}

La evaluación a los docentes puede ser utilizada como una herramienta de toma de decisiones para las instituciones de educación superior que estén en la búsqueda de una mejora continua del proceso de enseñanza-aprendizaje al considerar la percepción actual que tienen los alumnos siendo que estos son los receptores del servicio educativo que brinda la universidad y en conjunto con ella está interesado en recibir un servicio de calidad que le permita el día de mañana contar con competencias adecuadas para su desempeño profesional.
GAMBOA-CERDA, Silvia, VILLEGAS-FLORES, Ignacio, MEDELLÍN-RAMÍREZ, Juan Emilio y HERRERA-CHAIRES, María del Carmen. La evaluación docente. Una herramienta en la mejora del proceso enseñanza-aprendizaje en las instituciones de educación superior. Revista Teoría Educativa. 2019 


\section{Objetivos}

\section{Objetivo General}

Identificar la apreciación actual que tienen los alumnos de las materias de contabilidad básica de la licenciatura de Contador Público acerca del desempeño de sus docentes y las acciones desde la perspectiva de los autores que se deben tomar para que éste sea el más adecuado.

\section{Objetivos específicos}

- Dar retroalimentación al docente respecto de su desempeño frente a grupo según los resultados del instrumento de evaluación.

- Brindar a la institución de un análisis del instrumento de evaluación para la toma de decisiones encaminadas al fortalecimiento de las pareas de oportunidad detectadas.

- Analizar la coherencia y pertinencia de la percepción que tiene el alumno respecto de sus profesores.

\section{Marco Teórico}

Para la ANUIES (2006) la educación superior representa una alternativa de ensanchar las oportunidades vitales de los individuos y una vía que dispone la sociedad para reflexionar sobre sí misma en un entorno cada vez más complejo y dinámico. Asimismo, ANUIES destaca que la Educación Superior debe ofrecer a los jóvenes un espacio de formación para su desarrollo personal y profesional que valore la libertad, el desarrollo cultural y la cohesión social. Por lo tanto, la responsabilidad de la Educación Superior; es ofrecer una educación de calidad que contribuya a la formación del profesionista en un contexto global. En tal escenario, la misión de las Instituciones de Educación Superior consiste en cumplir con un papel de extrema importancia para favorecer el desarrollo del país y contribuir al bienestar de las personas.

Para Aponte-Hernández et al. (2008) el papel de la educación superior en estos tiempos es el de contribuir con el desarrollo de la sociedad del conocimiento y de las competencias y los servicios que se necesitan de acuerdo con el contexto de cada época.
Asimismo el sistema de educación superior mexicano está conformado por más de 1500 instituciones públicas y privadas, las cuales se clasifican en cuatro tipos: universidades, institutos tecnológicos, escuelas normales y universidades tecnológicas; conforme la clasificación de la Asociación Nacional de Universidades e Instituciones de Educación Superior (Luengo, 2003).

De acuerdo con lo anterior, las Instituciones de Educación Superior mantienen un papel importante en el desarrollo sostenido del país, es aquí en donde las personas se preparan para enfrentarse a un mercado laboral que demanda profesionistas competentes que se adapten a los diversos escenarios sociales; se desarrolle la perspectiva de una sociedad del conocimiento, sobre todo si pueden llevar a cabo cambios fundamentales en sus modelos de formación, de aprendizaje y de innovación. (Didriksson, Medina, Rojas, Bozzore y Hermo, 2008).

Para lograrlo se resalta la importancia de que los procesos educativos tengan una calidad socialmente aceptada, ya que el entorno actual señala un especial énfasis en los modelos eficientistas que privilegian la gestión institucional sobre el trabajo pedagógico y la investigación curricular. En este ámbito se establece un conjunto de competencias hacia cuyo desarrollo debe orientarse el trabajo escolar, y su evaluación se define en términos de conductas observables que pueden ser comparadas con estándares sociales previamente definidos como deseables y en correspondencia con patrones establecidos en el marco del sistema político, económico e ideológico hegemónico (Orozco Cruz, 2009).

Al hablar de evaluación educativa se debe considerar la de los profesores la cual debe llevarse a cabo como un proceso sistemático y metódico que, mediante instrumentos o procedimientos formales, recopile información sobre características seleccionadas de los docentes para juzgarlas de conformidad con indicadores previamente establecidos a fin de apoyar decisiones institucionales. Dicha evaluación se debe desarrollar con sentido metodológico y técnico para que los resultados que aporte sirvan para mejorar los perfiles formativos de los estudiantes (Gómez Bonilla 2009). 
También es importante mencionar que el gobierno mexicano en los últimos años ha buscado imponer la evaluación docente como una estrategia política para recuperar la rectoría del Estado en materia educativa, elevar la calidad de los servicios públicos de educación y establecer dispositivos administrativo-laborales de control sobre el ingreso, permanencia, promoción y reconocimiento en el Servicio Profesional Docente. (Guzmán Marín, 2018).

De acuerdo con la UASLP (2014) la evaluación de la docencia es una de las acciones cuyo objetivo es transparentar los procesos, prácticas y estado actual de la docencia en la institución.

Dentro de su modelo de competencias docentes los estudiantes de los diferentes programas académicos evalúan que sus profesores cumplan correctamente aspectos como la planeación y diseño del proceso de enseñanza- aprendizaje, proceso de enseñanza y aprendizaje, ambiente de aprendizaje, evaluación del aprendizaje, incorporación de tecnología, dominio de la materia y responsabilidad.

La utilidad e importancia de lo anterior se refleja en un reporte de evaluación docente que integra además de los resultados de su evaluación; una realimentación por cada una de las dimensiones comprendidas en la encuesta y recomendaciones para mejorar su práctica docente y por tanto los resultados de aprendizaje de los estudiantes.

Según la Facultad de Contaduría y Administración, FCA (2014) con la finalidad de enfrentar los requerimientos de una manera integral se ha trabajado en un enfoque basado en competencias por lo que sus programas educativos se estructuran actualmente en un semestre de integración y dos ciclos; el primero que ofrece tanto una formación básica como el desarrollo de competencias para el desempeño laboral y el segundo orientado a la formación profesional.

Asimismo, se contemplan cursos electivos libres diseñados con la intención de permitir el desarrollo de competencias adecuadas a las características de la demanda laboral.
Para la licenciatura de Contador Público las competencias que busca desarrollar en sus egresados van dirigidas hacía el diseño, desarrollo e implementación de sistemas de información del área de contabilidad, finanzas, costos, fiscal y auditoría incorporando la normatividad nacional e internacional, con el fin de maximizar el valor de la empresa de manera responsable y ética.

De acuerdo con el Programa de asignaturas para la licenciatura de Contador Público de Facultad de Contaduría y Administración, FCA (2015) dentro de las materias cursadas en el primer ciclo del área de Contabilidad Básica se encuentran las materias de Introducción a la Contabilidad, Estados Financieros, Bases de la Información Financiera y Normas Financieras.

\section{Metodología de investigación}

La metodología aplicada en esta investigación fue un diseño mixto de triangulación, con las variantes de convergencia y de transformación de datos. Para Creswell y Plano (2007) el modelo de convergencia representa un diseño de triangulación de métodos mixtos; en este modelo, el investigador recoge y analiza los datos cuantitativos de los cualitativos por separado comparando y poniendo en contraste los diversos resultados. Se trata de que el investigador compare y combine los dos conjuntos de datos relacionando por separado los resultados, pero en la interpretación los integre.

En la primera etapa se realizó el análisis cualitativo de diversos documentos relacionados con la evaluación docente, los planes de estudio y materias de la licenciatura de contador público que ofrece la Universidad Autónoma de San Luis Potosí y su Plan Institucional de Desarrollo. En la segunda etapa se analizaron los resultados de un instrumento de evaluación al que fue sometida la población sujeta del estudio.

La población participante en esta investigación fueron los 974 alumnos que cursaron materias de contabilidad básica de la mencionada licenciatura, de donde se tomó una muestra aleatoria simple de 927 estudiantes en la cual la selección de los elementos que la integraron y en donde cada uno de los elementos tuvo una probabilidad de selección conocida y equitativa (Malhotra, 2004, p. 325). 
En el diseño cualitativo se utilizó el método de recolección de documentos y materiales, por considerar que ayuda a entender el fenómeno central del estudio (Hernández, Fernández-Collado y Babtista, 2009) el cual permitió la revisión, comparación y codificación de los datos para tener una descripción más completa de los documentos. En el diseño cuantitativo se utilizó la estadística descriptiva, se utilizó el Statistical Package for the Social Sciences (SPSS $($ )), para dicho análisis. Para analizar los datos se llevaron a cabo los siguientes pasos:

1. Recopilación y análisis de diversas fuentes documentales y por Internet.

2. Los documentos recopilados fueron organizados para su análisis en diagramas y cuadros comparativos, con el propósito de fundamentar los resultados relacionados con las materias que cursaron y su evaluación docente.

3. Se realizó una clasificación y comparación de los resultados del instrumento de evaluación docente.

\section{Resultados}

La población participante en esta investigación son los 974 alumnos que cursaron alguna de las 4 materias de Contaduría Básica de la licenciatura en Contaduría Pública que ofrece la Universidad Autónoma de San Luis Potosí durante el semestre Enero-Junio 2019, de los cuales se tomó una muestra aleatoria simple de 927 alumnos de los 39 grupos en que se imparten estas asignaturas $y$ fueron sometidos al instrumento de evaluación institucional del cual se obtuvo la información correspondiente para analizar la percepción del desempeño de sus docentes en las materias anteriormente mencionadas.

Al hablar de 39 grupos es importante mencionar que se componen de 6 grupos de Introducción a la Contabilidad, 11 grupos de Estados Financieros, Bases de la Información Financiera y 11 grupos de Normas Financieras en donde se revisaron las competencias generales y por materia presentando en lo general una desviación estándar de 1.1488 y un lo particular una desviación que oscila entre 0.7117 y 1.3497 según se muestra en la Tabla 1.

\begin{tabular}{|l|r|r|}
\hline \multicolumn{1}{|c}{ Materia } & \multicolumn{1}{c|}{ Desv. Desviación } \\
\hline $\begin{array}{l}\text { Bases de la informacion } \\
\text { financiera }\end{array}$ & 11 & 1.2158 \\
\hline Estados financieros & 11 & 1.3497 \\
\hline $\begin{array}{l}\text { Introduccion a la } \\
\text { contabilidad }\end{array}$ & 6 & 1.3277 \\
\hline Normas financieras & 11 & 0.7117 \\
\hline Total & $\mathbf{3 9}$ & $\mathbf{1 . 1 4 8 8}$ \\
\hline
\end{tabular}

Tabla 1 Evaluación general de las competencias docentes por materia

Fuente: elaboración propia

A través del citado instrumento de evaluación es que la institución busca evaluar aspectos de primordial importancia que deben cumplir sus maestros para mantener los estándares de calidad educativa planteados en el Plan Institucional de Desarrollo 2013-2023 donde se evalúan aspectos generales y competencias docentes como planeación y diseño, conducción y evaluación del aprendizaje e incorporación de tecnología.

Derivado de lo anterior se pudo observar que los alumnos encuentran equilibrados los aspectos y competencias con las que deben contar sus docentes, ya que entre todas las variables hubo menos de 1 punto de diferencia media entre el indicador más alto y el más bajo. Como aspecto mejor evaluado en sus maestros de las materias de Contaduría Básica se encuentran los aspectos generales con una media de 7.96 puntos, seguido por el proceso enseñanza- aprendizaje donde obtuvieron 7.71 puntos. En tercer lugar hubo un empate en la planeación y diseño y el ambiente de aprendizaje ambos con 7.43 puntos. Los aspectos con ponderación media más baja fueron la evaluación general de competencias docentes y evaluación del aprendizaje con 7.36 y 7.31 puntos respectivamente y como el área de oportunidad más importante la incorporación de tecnología en su práctica docente obteniendo una calificación de 7.01 puntos siendo esta la más baja en toda la evaluación. Estas calificaciones junto con los demás aspectos evaluados se muestran a detalle en el Gráfico 1.
GAMBOA-CERDA, Silvia, VILLEGAS-FLORES, Ignacio, MEDELLÍN-RAMÍREZ, Juan Emilio y HERRERA-CHAIRES, María del Carmen. La evaluación docente. Una herramienta en la mejora del proceso enseñanza-aprendizaje en las instituciones de educación superior. Revista Teoría Educativa. 2019 


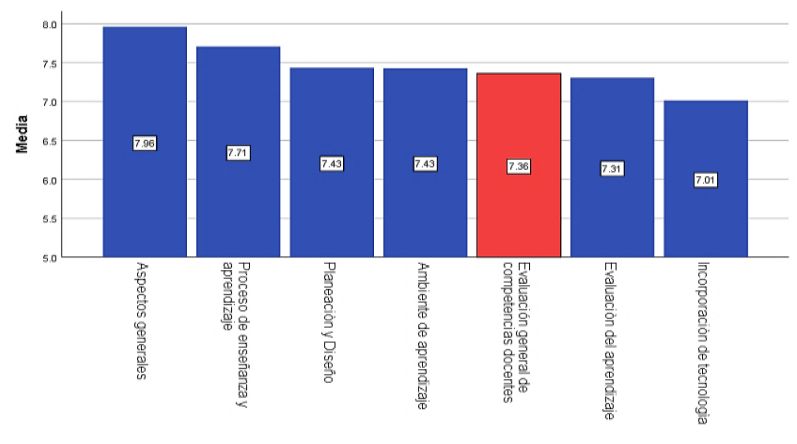

Gráfico 1 Evaluación general de docentes de las materias de contabilidad básica

Fuente: elaboración propia

Para complementar la información anterior es importante señalar que las valoraciones de los criterios mencionados fueron analizadas basados en las ponderaciones mínimas y máximas que obtuvo cada constructo en el instrumento de evaluación, con un margen de error muy bajo ya que todos los criterios presentan una desviación estándar muy cercana a la unidad. Dentro de los criterios evaluados se observó que los aspectos generales además de ser el criterio mejor evaluado según la media, también fue el que presentó la desviación estándar más baja con lo que se refuerza la confiabilidad en la determinación del aspecto más valorado de los docentes evaluados. La información pormenorizada de este análisis se detalla en la tabla 2.

\begin{tabular}{|c|c|c|c|c|}
\hline $\begin{array}{c}\text { Criterios o } \\
\text { constructos }\end{array}$ & Mínimo & Máximo & Media & $\begin{array}{c}\text { Desv. } \\
\text { Desviación }\end{array}$ \\
\hline $\begin{array}{l}\text { Aspectos } \\
\text { generales }\end{array}$ & 5.50 & 9.33 & 7.96 & 1.05169 \\
\hline $\begin{array}{lr}\text { Proceso } & \text { de } \\
\text { enseñanza } & \mathrm{y} \\
\text { aprendizaje } & \end{array}$ & 5.25 & 9.39 & 7.71 & 1.14872 \\
\hline $\begin{array}{l}\text { Planeación } \\
\text { Diseño }\end{array}$ & 4.38 & 9.04 & 7.43 & 1.24186 \\
\hline $\begin{array}{ll}\begin{array}{l}\text { Ambiente } \\
\text { aprendizaje }\end{array} & \text { de } \\
\end{array}$ & 3.75 & 9.72 & 7.43 & 1.29884 \\
\hline $\begin{array}{l}\text { Evaluación } \\
\text { general de } \\
\text { competencias } \\
\text { docentes }\end{array}$ & 4.7 & 9.3 & 7.36 & 1.1488 \\
\hline $\begin{array}{ll}\begin{array}{l}\text { Evaluación } \\
\text { aprendizaje }\end{array} & \text { del }\end{array}$ & 4.69 & 9.65 & 7.31 & 1.18737 \\
\hline $\begin{array}{ll}\text { Incorporación de } \\
\text { tecnología }\end{array}$ & 4.14 & 9.26 & 7.01 & 1.26871 \\
\hline
\end{tabular}

Tabla 2 Resultados por criterios evaluados Fuente: elaboración propia

Al hablar de los aspectos generales evaluados la institución presta atención a que el docente plantee el conocimiento de manera interesante y que se refleje en un buen nivel de aprendizaje por parte de los alumnos, demostrando buen dominio e ilación de teoría con la práctica, así como cuidando su puntualidad y asistencia a las horas clase establecidas.
Aunado a lo anterior surge la necesidad de que el docente planee y diseñe apropiadamente sus lecciones para lo cual debe siempre dejar claro el objetivo de aprendizaje de la clase, ligándolo con conocimientos previamente adquiridos por los estudiantes ya sea en la misma materia o en cursos anteriores y soportando toda la información brindada a los alumnos con la bibliografía sugerida la cual también debe verse reflejada en los materiales que prepare para impartir la materia.

En lo referente a la conducción del aprendizaje, la institución divide las competencias que deben poseer los docentes en el proceso de enseñanza- aprendizaje y en el ambiente de aprendizaje, siendo que para el proceso de enseñanza-aprendizaje buscan que el maestro sea capaz de que el alumno comprenda y aplique los conocimientos, a través de una explicación apropiada y actividades de aprendizaje para fortalecer lo visto en clase, así como el fomento en el estudiante del aprendizaje autónomo. Por otro lado en cuanto al ambiente de aprendizaje la universidad busca que el docente propicie sea el adecuado y promueva la cooperación entre los mismos alumnos para un mejor aprendizaje.

Para lograr una práctica docente con estándares de calidad es que se busca que el docente esté actualizado en materia de innovación educativa e implemente en sus clases la incorporación de la tecnología tanto en los materiales que diseñe para dar sus clases así como en las actividades que pida a los alumnos desarrollen para que ellos también busquen y produzcan materiales vanguardistas que les puedan servir para su práctica profesional.

Dentro del desempeño del docente se encuentra la función que tiene el maestro de evaluar a sus estudiantes, lo cual deberá lograr a través de la implementación de diferentes instrumentos de evaluación, previamente anunciados a sus estudiantes que le permita darles resultados oportunos con una retroalimentación tanto para el docente como para el alumno de los conocimientos adquiridos y de los que aún se deben fortalecer. 
Todos estos componentes específicos que en su conjunto integran los criterios generales evaluados fueron sometidos a análisis bajo la aplicación de los mismos instrumentos de medición, donde se pudo observar que el componente más valorado por los estudiantes es el interés que el docente logra captar durante sus clases, mientras que preocupantemente el componente específico con la ponderación más baja fue el nivel de aprendizaje que obtienen los alumnos en estas asignaturas.

El resultado del análisis individualizado de los conceptos específicos se muestra en la tabla 3.

\begin{tabular}{|l|r|r|r|r|}
\hline \multicolumn{1}{|c}{ Componentes } & Mínimo & \multicolumn{1}{c|}{ Máximo } & \multicolumn{1}{c|}{ Media } & \multicolumn{1}{c|}{$\begin{array}{c}\text { Desv. } \\
\text { Desviación }\end{array}$} \\
\hline InteresanteAG & 6.87 & 10.00 & 8.8479 & 0.67378 \\
\hline Dominio teoría-práctica & 5.62 & 9.82 & 8.3287 & 1.09307 \\
\hline Aplicación conocimiento & 6.02 & 9.69 & 8.0636 & 1.07662 \\
\hline Asistencia & 5.00 & 10.00 & 7.9687 & 1.46939 \\
\hline Puntualidad & 4.65 & 10.00 & 7.8744 & 1.46052 \\
\hline Actividades de aprendizaje & 5.00 & 9.22 & 7.7154 & 1.10678 \\
\hline Comprensión contenidos & 5.62 & 9.44 & 7.6690 & 1.16631 \\
\hline Objetivo aprendizaje & 5.00 & 9.47 & 7.5782 & 1.24518 \\
\hline Aprendizaje autónomo & 5.00 & 9.44 & 7.5597 & 1.25831 \\
\hline Preparación Clases & 3.75 & 9.44 & 7.5372 & 1.42931 \\
\hline Retroalimentaciòn & 3.75 & 9.72 & 7.5310 & 1.28255 \\
\hline Explica claramente & 3.75 & 9.44 & 7.5221 & 1.37930 \\
\hline Comunica evaluación & 3.75 & 9.72 & 7.5087 & 1.37367 \\
\hline Buen ambiente & 3.75 & 9.72 & 7.4349 & 1.40840 \\
\hline Promueve colaboración & 3.75 & 9.72 & 7.4213 & 1.20777 \\
\hline $\begin{array}{l}\text { Evaluación general } \\
\text { competencias docentes }\end{array}$ & 4.7 & 9.3 & 7.359 & 1.1488 \\
\hline Conocimientos previos & 5.00 & 8.92 & 7.3115 & 1.08388 \\
\hline Bibliografía & 3.75 & 9.17 & 7.3087 & 1.38524 \\
\hline Comunicación TIC & 4.12 & 9.44 & 7.1708 & 1.39736 \\
\hline Resultados oportunos & 3.75 & 9.72 & 7.1126 & 1.31041 \\
\hline Evaluación diversificada & 3.75 & 9.44 & 7.0713 & 1.26299 \\
\hline Materiales diversos & 4.34 & 9.17 & 6.9808 & 1.18091 \\
\hline Búsqueda de información & 3.75 & 9.44 & 6.8890 & 1.40442 \\
\hline Nivel aprendizaje & 2.50 & 8.27 & 6.7695 & 1.25200 \\
\hline
\end{tabular}

Tabla 3 Análisis de los criterios específicos evaluados Fuente: elaboración propia

Para explicar de una manera más detallada y confiable los componentes específicos que integran cada uno de los criterios generales que la institución busca que los alumnos evalúen de sus docentes se realizó un análisis detallado de éstos a través de la determinación aritmética del alfa de cronbach, obteniendo la media de las correlaciones entre las variables que forman parte de la escala y confirmando con ello que el instrumento de evaluación es una herramienta real y puede ser utilizada por la institución para la toma de decisiones enfocadas al fortalecimiento de las competencias docentes con mayor área de oportunidad ya que los resultados de lo anterior reflejan que todos los elementos evaluados por el instrumento covarían entre sí y por ende se tiene una herramienta confiable. Los detalles se exponen en la tabla 4.

\begin{tabular}{|l|c|c|c|}
\hline \multicolumn{1}{|c}{ Criterio } & \multicolumn{1}{c}{$\begin{array}{c}\text { Varianza de } \\
\text { escala si el } \\
\text { elemento se ha } \\
\text { suprimido }\end{array}$} & $\begin{array}{c}\text { Correlación } \\
\text { total de } \\
\text { elementos } \\
\text { corregida }\end{array}$ & $\begin{array}{c}\text { Alfa de } \\
\text { Cronbach si el } \\
\text { elemento se ha } \\
\text { suprimido }\end{array}$ \\
\hline InteresanteAG & 638.975 & 0.501 & 0.988 \\
\hline Nivel aprendizaje & 602.146 & 0.859 & 0.986 \\
\hline $\begin{array}{l}\text { Dominio teoría- } \\
\text { práctica }\end{array}$ & 608.626 & 0.865 & 0.986 \\
\hline Asistencia & 596.635 & 0.804 & 0.986 \\
\hline Puntualidad & 593.629 & 0.853 & 0.986 \\
\hline $\begin{array}{l}\text { Conocimientos } \\
\text { previos }\end{array}$ & 606.453 & 0.915 & 0.986 \\
\hline $\begin{array}{l}\text { Objetivo } \\
\text { aprendizaje }\end{array}$ & 599.541 & 0.909 & 0.986 \\
\hline Preparación Clases & 587.968 & 0.959 & 0.985 \\
\hline Bibliografì & 592.345 & 0.923 & 0.986 \\
\hline $\begin{array}{l}\text { Comprensión } \\
\text { contenidos }\end{array}$ & 600.951 & 0.948 & 0.985 \\
\hline $\begin{array}{l}\text { Aplicación } \\
\text { conocimiento }\end{array}$ & 604.511 & 0.960 & 0.985 \\
\hline $\begin{array}{l}\text { Actividades } \\
\text { aprendizaje }\end{array}$ & 603.753 & 0.947 & 0.985 \\
\hline Explica claramente & 596.464 & 0.863 & 0.986 \\
\hline $\begin{array}{l}\text { Aprendizaje } \\
\text { autónomo }\end{array}$ & 595.695 & 0.964 & 0.985 \\
\hline Buen ambiente & 590.891 & 0.929 & 0.985 \\
\hline $\begin{array}{l}\text { Promueve } \\
\text { colaboración }\end{array}$ & 600.340 & 0.924 & 0.986 \\
\hline $\begin{array}{l}\text { Comunica } \\
\text { evaluación }\end{array}$ & 591.935 & 0.938 & 0.985 \\
\hline Retroalimentaciòn & 594.313 & 0.968 & 0.985 \\
\hline $\begin{array}{l}\text { Evaluación } \\
\text { diversificada }\end{array}$ & 599.780 & 0.891 & 0.986 \\
\hline $\begin{array}{l}\text { Resultados } \\
\text { oportunos }\end{array}$ & 610.689 & 0.681 & 0.987 \\
\hline Materiales diversos & 612.982 & 0.720 & 0.987 \\
\hline Comunicación TIC & 600.397 & 0.791 & 0.986 \\
\hline $\begin{array}{l}\text { Búsqueda } \\
\text { información }\end{array}$ & 594.392 & 0.878 & \\
\hline & & & \\
\hline
\end{tabular}

Tabla 4 Confiabilidad de los criterios específicos evaluados

Fuente: elaboración propia

Las competencias mencionadas anteriormente también se analizaron de forma general y particular por cada uno de los 39 grupos sujetos de este estudio, ya que se observó que es subjetivo el hablar de una ponderación por materia, siendo que una misma asignatura puede estar alta o bajamente evaluada dependiendo del docente que la imparte y la cantidad de alumnos que la cursan entre otros factores, por lo que el análisis de la evaluación general de competencias reflejó resultados que oscilaron entre 9.3 de la materia mejor evaluada a 4.7 puntos obtenido por la asignatura con más baja calificación, por lo que en la tabla 5 se muestra este análisis pormenorizado por grupo.
GAMBOA-CERDA, Silvia, VILLEGAS-FLORES, Ignacio, MEDELLÍN-RAMÍREZ, Juan Emilio y HERRERA-CHAIRES, María del Carmen. La evaluación docente. Una herramienta en la mejora del proceso enseñanza-aprendizaje en las instituciones de educación superior. Revista Teoría Educativa. 2019 


\begin{tabular}{|c|c|c|c|c|}
\hline Grupo & Semestre & Materia & $\begin{array}{c}\text { Evaluación } \\
\text { general de } \\
\text { competencias } \\
\text { docentes }\end{array}$ & Inscripción \\
\hline 20 & 2 & $\begin{array}{l}\text { ESTADOS } \\
\text { FINANCIEROS }\end{array}$ & 9.3 & 9 \\
\hline 28 & 2 & $\begin{array}{l}\text { ESTADOS } \\
\text { FINANCIEROS }\end{array}$ & 8.8 & 41 \\
\hline 3 & 1 & $\begin{array}{l}\text { INTRODUCCION A LA } \\
\text { CONTABILIDAD }\end{array}$ & 8.6 & 41 \\
\hline 13 & 1 & $\begin{array}{lcr}\text { BASES } & \text { DE } & \text { LA } \\
\text { INFORMACION } & \\
\text { FINANCIERA } & \\
\end{array}$ & 8.6 & 34 \\
\hline 9 & 1 & $\begin{array}{lcr}\text { BASES } & \text { DE } & \text { LA } \\
\text { INFORMACION } & \\
\text { FINANCIERA } & \\
\end{array}$ & 8.5 & 42 \\
\hline 15 & 1 & $\begin{array}{lcr}\text { BASES } & \text { DE } & \text { LA } \\
\text { INFORMACION } & \\
\text { FINANCIERA } & \end{array}$ & 8.5 & 4 \\
\hline 32 & 2 & $\begin{array}{l}\text { NORMAS } \\
\text { FINANCIERAS }\end{array}$ & 8.5 & 42 \\
\hline 2 & 1 & $\begin{array}{l}\text { INTRODUCCION A LA } \\
\text { CONTABILIDAD }\end{array}$ & 8.4 & 39 \\
\hline 33 & 2 & $\begin{array}{l}\text { NORMAS } \\
\text { FINANCIERAS }\end{array}$ & 8.4 & 27 \\
\hline 1 & 1 & $\begin{array}{l}\text { INTRODUCCION A LA } \\
\text { CONTABILIDAD }\end{array}$ & 8.3 & 40 \\
\hline 31 & 2 & $\begin{array}{l}\text { NORMAS } \\
\text { FINANCIERAS }\end{array}$ & 8.2 & 40 \\
\hline 10 & 1 & $\begin{array}{lcr}\text { BASES } & \text { DE } & \text { LA } \\
\text { INFORMACION } & \\
\text { FINANCIERA } & \\
\end{array}$ & 8.1 & 10 \\
\hline 17 & 1 & $\begin{array}{l}\text { NORMAS } \\
\text { FINANCIERAS }\end{array}$ & 8.1 & 39 \\
\hline 21 & 2 & $\begin{array}{l}\text { ESTADOS } \\
\text { FINANCIEROS }\end{array}$ & 8.1 & 35 \\
\hline 35 & 2 & $\begin{array}{l}\text { NORMAS } \\
\text { FINANCIERAS }\end{array}$ & 8.1 & 28 \\
\hline 37 & 2 & $\begin{array}{l}\text { NORMAS } \\
\text { FINANCIERAS } \\
\end{array}$ & 8.1 & 25 \\
\hline 14 & 1 & $\begin{array}{lcr}\text { BASES } & \text { DE } & \text { LA } \\
\text { INFORMACION } & \\
\text { FINANCIERA } & \\
\end{array}$ & 8.0 & 33 \\
\hline 8 & 1 & $\begin{array}{lcl}\text { BASES } & \text { DE } & \text { LA } \\
\text { INFORMACION } & \\
\text { FINANCIERA } & \\
\end{array}$ & 7.8 & 19 \\
\hline 27 & 2 & $\begin{array}{l}\text { ESTADOS } \\
\text { FINANCIEROS } \\
\end{array}$ & 7.8 & 41 \\
\hline 36 & 2 & $\begin{array}{l}\text { NORMAS } \\
\text { FINANCIERAS }\end{array}$ & 7.7 & 31 \\
\hline 11 & 1 & $\begin{array}{lcl}\text { BASES } & \text { DE } & \text { LA } \\
\text { INFORMACION } & \\
\text { FINANCIERA } & \\
\end{array}$ & 7.6 & 8 \\
\hline 34 & 2 & $\begin{array}{l}\text { NORMAS } \\
\text { FINANCIERAS }\end{array}$ & 7.6 & 3 \\
\hline 38 & 2 & $\begin{array}{l}\text { NORMAS } \\
\text { FINANCIERAS }\end{array}$ & 7.3 & 17 \\
\hline 29 & 2 & $\begin{array}{lcr}\text { BASES DE } & \text { LA } \\
\text { INFORMACION } & \\
\text { FINANCIERA } & \\
\end{array}$ & 7.1 & 37 \\
\hline 39 & 2 & $\begin{array}{l}\text { NORMAS } \\
\text { FINANCIERAS }\end{array}$ & 7.0 & 8 \\
\hline 12 & 1 & $\begin{array}{lcr}\text { BASES DE } & \text { LA } \\
\text { INFORMACION } & \\
\text { FINANCIERA } & \end{array}$ & 6.9 & 21 \\
\hline 19 & 2 & $\begin{array}{l}\text { ESTADOS } \\
\text { FINANCIEROS }\end{array}$ & 6.9 & 28 \\
\hline 23 & 2 & $\begin{array}{l}\text { ESTADOS } \\
\text { FINANCIEROS }\end{array}$ & 6.6 & 18 \\
\hline 22 & 2 & $\begin{array}{l}\text { ESTADOS } \\
\text { FINANCIEROS }\end{array}$ & 6.4 & 9 \\
\hline 5 & 1 & $\begin{array}{l}\text { INTRODUCCION A LA } \\
\text { CONTABILIDAD }\end{array}$ & 6.3 & 36 \\
\hline 7 & 1 & $\begin{array}{l}\text { ESTADOS } \\
\text { FINANCIEROS }\end{array}$ & 6.2 & 12 \\
\hline 18 & 1 & $\begin{array}{l}\text { NORMAS } \\
\text { FINANCIERAS }\end{array}$ & 6.1 & 7 \\
\hline 26 & 2 & $\begin{array}{l}\text { ESTADOS } \\
\text { FINANCIEROS }\end{array}$ & 6.1 & 23 \\
\hline 4 & 1 & $\begin{array}{l}\text { INTRODUCCION A LA } \\
\text { CONTABILIDAD }\end{array}$ & 6.0 & 40 \\
\hline 16 & 1 & $\begin{array}{ll}\text { BASES DE } & \text { LA } \\
\text { INFORMACION } & \\
\text { FINANCIERA } & \end{array}$ & 5.9 & 6 \\
\hline 6 & 1 & $\begin{array}{l}\text { INTRODUCCION A LA } \\
\text { CONTABILIDAD }\end{array}$ & 5.8 & 37 \\
\hline 24 & 2 & $\begin{array}{l}\text { ESTADOS } \\
\text { FINANCIEROS }\end{array}$ & 5.5 & 7 \\
\hline 25 & 2 & $\begin{array}{l}\text { ESTADOS } \\
\text { FINANCIEROS }\end{array}$ & 5.1 & 35 \\
\hline 30 & 2 & $\begin{array}{lcr}\text { BASES } & \text { DE } & \text { LA } \\
\text { INFORMACION } & \\
\text { FINANCIERA } & \\
\end{array}$ & 4.7 & 2 \\
\hline Гotal & 39 & & & 974 \\
\hline
\end{tabular}

Tabla 5 Evaluación general de competencias docentes por grupo

Fuente: elaboración propia

\section{Conclusiones}

La evaluación docente debe de ser considerada como una herramienta en primera instancia para que el maestro sea capaz de responder a las necesidades actuales de formar profesionistas competentes y a su vez identifique la perspectiva que los alumnos ven en ellos y de manera continua se estén reinventando para dar la mejor versión de sí mismos; y por otro lado también debe de servir a las instituciones educativas para tomar acciones basadas en este criterio y fomentar actividades de capacitación y seguimiento puntual a sus docentes para que desempeñen el mejor papel posible frente a grupo.

La realidad es que hoy en nuestro país se continua tomando más en cuenta la evaluación al alumno y no al docente, ya sea por factores políticos y/o sociales, pero una evaluación docente bien aplicada puede dar grandes resultados que beneficiaran a la sociedad a corto y largo plazo, motivo por el cual esta investigación a través de un estudio particular pudo identificar que hoy en día los alumnos valoran primordialmente el tener lecciones interesantes que les dejen un conocimiento significativo y práctico, a pesar de que el nivel de aprendizaje lo tomen como un criterio poco valorado.

Se observó que dentro de los criterios evaluados la institución concuerda con lo que los alumnos valoran, ya que busca que el docente plantee el conocimiento de manera interesante pero difiere con ellos en cuanto al nivel de aprendizaje ya que para la universidad es un indicador de educación cualitativa, por lo que debe fomentar que sus maestros presenten buen dominio e ilación de teoría con la práctica, así como cuidando su puntualidad y asistencia a las horas clase establecidas, siendo que estos son estándares que deben de transmitir a sus estudiantes para que el día que estos se encuentren en el ámbito laboral puedan hacerlo de forma integral. Es importante resaltar que los resultados de una evaluación docente son sujetos a analizar más variables que la ponderación misma, ya que las competencias evaluadas pueden cambiar dependiendo del docente que imparte una misma asignatura, la cantidad de alumnos que la cursan, el diseño del plan de estudios entre otros factores. 
Los resultados que arroja este tipo de instrumento de evaluación le permite a la universidad obtener de forma sistemática la percepción que tienen los alumnos de sus docentes, conocer sus fortalezas y áreas de oportunidad, el cumplimiento adecuado de los planes de estudio y las herramientas didácticas y tecnológicas que utilizan para transmitir su conocimiento con una ponderación cierta. Tanto las fortalezas como las áreas de oportunidad detectadas en la evaluación le permitirán la mejora continua del proceso educativo.

Derivado de lo anterior el tema de esta investigación sienta un precedente que puede ser estudiado a fondo al analizar otros factores que influencian el proceso enseñanza-aprendizaje, tales como socioeconómicos, culturales y educativos, con la finalidad de comprobar su influencia en la formación de futuros profesionales, además de que la población estudio de esta investigación fue muy delimitada, pero esto se puede extender a más materias de la misma licenciatura e inclusive a otros programas académicos.

\section{Referencias}

Asociación Nacional de Universidades e Instituciones de Educación Superior. (2006). Consolidación y avance de la educación superior en México. Distrito Federal, México.

Aponte-Hernández, E., Mendes, M., Psicoya, B., Celton, D. y Macador, D. (2008). Desigualdad, inclusión y equidad en a educación superior en América Latina y el Caribe: Tendencias y Escenario Alternativo en el Horizonte 2011.

Creswell W., y Plano Clark, V, L. (2007). Designing and conducting mixed methods research. Thousand Oaks, CA: Sage Publications.

Didriksson, A., Medina, E., Rojas, M., Bozzozero, L., Hermo, J. (2008). Contexto global y regional de la educación superior en América Latina y el Caribe. En: Tendencias de la Educacion Superior en America Latina y el Caribe / editado por Ana Lucia Gazzola y Axel Didriksson.- Caracas: IESALC-UNESCO, 2008.

Facultad de Contaduría y Administración, FCA (2014). Plan de desarrollo 2014-2023 Facultad de Contaduría y Administración.
Facultad de Contaduría y Administración, FCA (2015). Programa de asignaturas 2015 Contador Público. Recuperado el 24 de agosto 2019 de: http://www.fca.uaslp.mx/Paginas/oferta\%20edu cativa/contador\%20publico/Programa-de-

Asignaturas-de-CP-2015.aspx

Gómez Bonilla, E., Gómez Bonilla, Y. (2009). La evaluación de la docencia basada en la opinión de los alumnos: el perfil docente en la licenciatura en Historia.

Guzmán Marín, F. (2018). La Experiencia de la Evaluación Docente en México: Análisis Crítico de la Imposición del Servicio.

Hernández, R., Fernández-Collado, C. y Babtista, P. (2009). Metodología de la Investigación. (4 ${ }^{a}$. Ed). México: McGraw-Hill.

Luengo, E. (2003). Tendencias de la Educación Superior en México: Una lectura desde la perspectiva de la Complejidad. México: ANUIES. Recuperado de: $\mathrm{http}: / /$ yadiramendozareyes.files.wordpress.com/ 2013/03/tendencias-de-la-educacic3b3nsuperior-en-mc3a9xico.pdf

Malhotra, K. (2004). Investigación de mercados. Distrito Federal, México: Pearson Education.

Orozco Cruz, J (2009). ¿Calidad de la educación o educación de calidad? Una preocupación más allá del mercado. Revista Iberoamericana de Educación.

Universidad Autónoma de San Luis Potosí (2014). Plan Institucional de Desarrollo 20132023.
GAMBOA-CERDA, Silvia, VILLEGAS-FLORES, Ignacio, MEDELLÍN-RAMÍREZ, Juan Emilio y HERRERA-CHAIRES, María del Carmen. La evaluación docente. Una herramienta en la mejora del proceso enseñanza-aprendizaje en las instituciones de educación superior. Revista Teoría Educativa. 2019 